\title{
Corrigenda: \\ On the product theory of singular integrals
}

Rev. Mat. Iberoamericana 20 (2004), no. 2, 531-561.

\section{Alexander Nagel and Elias M. Stein}

We wish to acknowledge and correct an error $^{1}$ in a proof in our paper On the product theory of singular integrals, which appeared in Revista Matemática Iberoamericana, volume 20, number 2, 2004, pages 531-561. In Lemma 2.3.2, part (a), we wish to show that for $\lambda>0$, the operator $R(\lambda, \mathcal{L})=(\lambda I+\mathcal{L})^{-1}$ is bounded on $L^{\infty}(M)$ with a norm that may depend on $\lambda$. We write the operator as

$$
R(\lambda, \mathcal{L})[f](x)=\int_{M} f(y) r_{\lambda}(x, y) d y .
$$

It then follows that

$$
r_{\lambda}(x, y)=\int_{0}^{\infty} e^{-\lambda s} H(s, x, y) d s
$$

where $H(s, x, y)$ is the heat kernel for the operator $\mathcal{L}$. We assert in equation (2.12) that there is a constant $C$ so that for all $\lambda>0$

$$
\left|r_{\lambda}(x, y)\right| \leq C \frac{d^{2}(x, y)}{V(x, y)}
$$

This is not correct when $M$ is a compact manifold. It should be replaced by the statement that when $M$ is compact and of dimension at least 3 , there is a constant $C$ so that for all $\lambda>0$

$$
\left|r_{\lambda}(x, y)\right| \leq C \frac{d^{2}(x, y)}{V(x, y)}+\frac{C}{\lambda} .
$$

This estimate still shows that the operator $R(\lambda, \mathcal{L})$ is bounded on $L^{\infty}(M)$ if $M$ is compact.

\footnotetext{
${ }^{1}$ We wish to thank Professor Dachun Yang of Beijing Normal University for bringing this error to our attention.
} 
The error in our proof occurs on page 539 in the estimation of the integral for $r_{\lambda}(x, y)$. We do not use the decay given by $e^{-\lambda s}$ and assert that one part

$$
\int_{d(x, y)^{2}}^{\infty} e^{-\lambda s}|H(s, x, y)| d s \lesssim \int_{d(x, y)^{2}}^{\infty} V_{\sqrt{s}}(x)^{-1} d s
$$

arises only in the case of noncompact $M$. This is not true, and one must deal with this integral in all cases. However we can keep the decay term and write

$$
\int_{d(x, y)^{2}}^{\infty} e^{-\lambda s}|H(s, x, y)| d s \lesssim \int_{d(x, y)^{2}}^{1} V_{\sqrt{s}}(x)^{-1} d s+\int_{1}^{\infty} e^{-\lambda s} d s .
$$

The first term on the right is handled as in the non-compact case, using the estimate

$$
V_{d(x, y) t}(x) \geq t^{3} V(x, y),
$$

which is valid when $t>1$ and $t d(x, y) \leq 1$. The second term on the right gives $C \lambda^{-1}$.

Recibido: 6 de abril de 2005

Alexander Nagel

Department of Mathematics

University of Wisconsin

Madison, WI 53706-1388, USA

nagel@math . wisc . edu

Elias M. Stein

Department of Mathematics

Princeton University

Princeton, NJ 08544, USA

stein@math.princeton.edu 